\title{
Novel polyamine-based Histone deacetylases-Lysine demethylase 1 dual binding inhibitors
}

\author{
Andrea Milelli ${ }^{\mathrm{a}, *}$, Chiara Marchetti ${ }^{\mathrm{b}}$, Eleonora Turrini ${ }^{\mathrm{a}}$, Elena Catanzaro ${ }^{\mathrm{a}}$, Roberta Mazzone ${ }^{\mathrm{c}}$, \\ Daniela Tomaselli ${ }^{c}$, Carmela Fimognari ${ }^{a}$, Vincenzo Tumiatti ${ }^{a}$, Anna Minarini ${ }^{b}$ \\ a Department for Life Quality Studies, Alma Mater Studiorum-University of Bologna, Corso d'Augusto 237, 47921 Rimini, Italy \\ ${ }^{\mathrm{b}}$ Department of Pharmacy and Biotechnology, Alma Mater Studiorum-University of Bologna, Via Belmeloro 6, 40126 Bologna, Italy \\ ${ }^{\mathrm{c}}$ Department of Drug Chemistry and Technologies, Sapienza University of Rome, P.le Aldo Moro 5, 00185 Roma, Italy
}

\section{A R T I C L E I N F O}

\section{Article history:}

Received 29 November 2017

Revised 15 February 2018

Accepted 16 February 2018

Available online 17 February 2018

Keywords:

Dual-binding agents

Epigenetics

Histone deacetylase inhibitors

Lysine demethylase inhibitors

Polyamines

\begin{abstract}
A B S T R A C T
Epigenetic modulators Histone deacetylases (HDACs) and Lysine demethylase (LSD1) are validated targets for anticancer therapy. Both HDAC1/2 and LSD1 are found in association with the repressor protein CoREST in a transcriptional co-repressor complex, which is responsible for gene silencing. Combined modulation of both targets results in a synergistic antiproliferative activity. In the present investigation, we report about the design and synthesis of a series of polyamine-based HDACs-LSD1 dual binding inhibitors obtained by coupling Vorinostat and Tranylcypromine. Compound $\mathbf{4}$ emerged as the most promising of the synthesized series, showing good inhibitory activity towards HDAC1 and LSD1 either in vitro and in cell-based assay ( $\mathrm{Ki}=42.52 \pm 8.94 \mathrm{nM}$ and $\mathrm{IC}_{50}=3.85 \mu \mathrm{M}$, respectively). Furthermore, at 70.0 $\mu \mathrm{M}$ compound 4 induced a more pronounced cytotoxic effect than Vorinostat (68.6\% vs $56.6 \%$ of dead cells) in MCF7 cancer cell line.
\end{abstract}

(c) 2018 Elsevier Ltd. All rights reserved.
Cancer is a multifactorial disease whose onset derives from both genetic and epigenetic events; in recent years, epigenetic has gained notable consideration and epigenetic targets have been validated in therapy. ${ }^{1}$ Epigenetic changes are reversible chromatin modifications that lead to activation or suppression of gene expressions. The epigenetic control of gene expressions takes place through modification of the DNA itself, i.e. methylation, or DNAassociated proteins, i.e. methylation and acetylation. According to the subject and the type of modification, chromatin can adopt conformational changes that lead to the activation or suppression of gene expression. ${ }^{2}$ Histone deacetylases (HDACs) are one of the most important classes of epigenetic enzymes responsible for removing acetyl groups from histone tails, determining a more closed, transcriptionally repressed, chromatin structure. ${ }^{3}$ Abnormal HDACs activity has been found to be associated with the aberrant gene expression and the development of several kinds of cancer. ${ }^{4}$ Inhibition of HDACs activities restore normal gene expression resulting in cell cycle arrest, apoptotic cell death and cell differentiation. ${ }^{5}$ Different HDAC inhibitors (HDACis) have been approved by the FDA, i.e. vorinostat, romidepsin, panobinostat and belinostat, and chidamide in China, and many others are

\footnotetext{
* Corresponding author.

E-mail address: andrea.milelli3@unibo.it (A. Milelli).
}

currently under evaluation in clinical trials (Fig. 1 ). ${ }^{6}$ Over the years, several different classes of HDACis have been identified; ${ }^{7}$ among them hydroxamate-based compounds hold a special interest in spite of their unfavourable ADMET properties. ${ }^{8}$ Hydroxamatebased inhibitors, among the most potent but rather unselective HDACis, bear the hydroxamic acid group able to strongly coordinate the $\mathrm{Zn}^{2+}$ located in the active site of the enzyme. The general structure for hydroxamate-HDACis comprises three main motifs: a $\mathrm{Zn}^{2+}$ binding group, a hydrophobic spacer and a cap group able to interact with the surface of the enzyme. Several clinical evidences reported that HDACis might be more effective as antiproliferative agents when used in combination with other chemotherapeutic or epigenetic drugs. For instance, HDACis potentiate the antiproliferative activities of Topoisomerase I and II inhibitors; ${ }^{9,10}$ further, HDACis in combination with the EGFR inhibitor Gefitinib synergistically induce growth inhibition and apoptosis induction in gefitinib-resistant cancer cell lines. ${ }^{11,12}$ Recently, Bhalla and coworkers reported that combined inhibition of HDACs and Lysine demethylase LSD1 is lethal in human AML cells. ${ }^{13}$ Furthermore, the same combination resulted in a synergistic increase of apoptotic cell death in glioblastoma multiforme cell line. ${ }^{14}$ LSD1 is a histone demethylase, member of the greater amine oxidase superfamily, that is responsible, among other activities, for the specific demethylation of mono- and dimethylated histone H3-Lys4. ${ }^{15}$ 


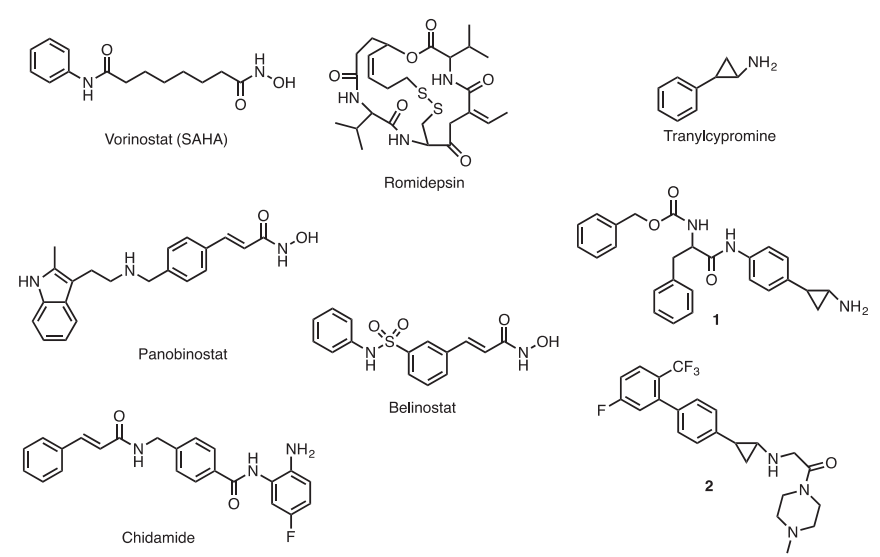

Fig. 1. Structures of known HDACis, Vorinostat, Romidepsin, Panobinostat, Belinostat and Chidamide, and LSD1 inhibitors, Tranylcypromine, $\mathbf{1}$ and $\mathbf{2}$.

LSD1 is overexpressed in various cancers and binds to several transcription factors, regulating the expression of a vast array of genes. ${ }^{16}$ LSD1 is found in association with the repressor protein CoREST and HDAC $1 / 2$, in a transcriptional co-repressor complex that is responsible for gene silencing. ${ }^{17}$ Most of the known LSD1 inhibitors developed share structural features with monoamine oxidase inhibitors. In particular, Tranylcypromine is a known irreversible LSD1 inhibitor and several Tranylcypromine-based derivatives have been reported so far (Fig. 1). The synergistic activity obtained combining HDACs and LSD1 inhibitors together with the finding that LSD1 is associated with CoREST and HDAC1/2 provided the rational for designing dual binding agents able to simultaneously modulate HDACs and LSD1. Herein, we report the design, the synthesis and the preliminary biological evaluation of a series of polyamine-based HDAC/LSD1 dual binding inhibitors.

The design of these new compounds began with the observation that various substituents may be introduced at the para position of the aromatic ring of both Vorinostat and Tranylcypromine without a dramatic drop in the inhibitory activity. The two pharmacophores have been linked through a polyamine chain since it has been previously demonstrated that a) protonated nitrogen atoms can establish interactions with negatively charged amino acids $^{18,19}$ and b) polyamines interact with both HDACs and LSD1 proteins. $^{20-22}$ Three different polyamine chains have been chosen differing in the number of the nitrogen atoms and in the distance between them; indeed, compound $\mathbf{3}$ has a spermidine-like linker (3-3), compound 4 has a spermine linker (3-4-3) and compound 5 carries a spermine-like connecting unit characterized by longer (6-8-6) polymethylenes chain between the nitrogen atoms (Fig. 2).

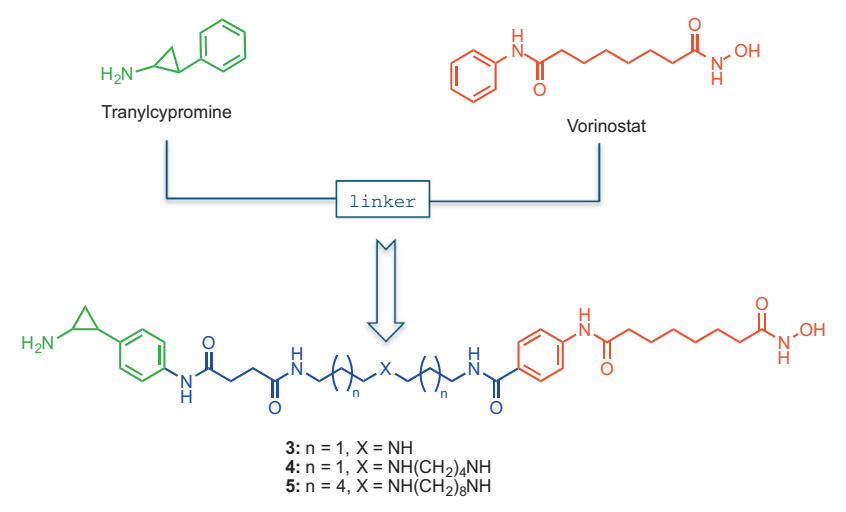

Fig. 2. Drug design leading to dual binding agents 3-5.
During the drafting of this manuscript, two papers reporting about novel dual HDAC-LSD1 inhibitors appeared. ${ }^{23,24}$ However, these compounds turned out to be structurally slightly different than those reported in the present manuscript.

For the synthesis of target compounds 3-5 a stepwise linear synthetic approach was developed (Scheme 1). Suberic anhydride 7 was condensed with methyl 4-aminobenzoate 6 to generate the corresponding acid $\mathbf{8}$. This was coupled to O-(Tetrahydro- $2 \mathrm{H}-$ pyran-2-yl)hydroxylamine in order to obtain the protected hydroxamic acid 9, using EDC as coupling agent. Basic hydrolysis of the methyl ester of $\mathbf{9}$ furnished the carboxylic acid $\mathbf{1 0}$ that was coupled with the suitable protected polyamines 11-13. ${ }^{25,26}$ Many efforts were carried out in order to optimize the coupling conditions of 10 with 11-13: after variation of solvents (DCM, DMF, THF), reaction times and coupling auxiliary (EtOCOCl and $\mathrm{Et}_{3} \mathrm{~N}, \mathrm{IBCF}$ and $\mathrm{Et}_{3} \mathrm{~N}, \mathrm{DCC}$ and HOBt, DCC and DMAP, EDC and HOBt) it was found that the use of EDC and DMAP gave the best yields. Basic deprotection of the trifluoromethyl protecting group led to the primary amines $\mathbf{1 7 - 1 9}$, which further reacted with succinic anhydride leading to the corresponding acids 20-22. Coupling of the latter with Boc-protected tranylcypromine ${ }^{27}$ generated the fully-protected dimers 24-26. Also in this case, several reaction conditions were evaluated with the aim to optimize the coupling reaction and again better yields were obtained using EDC as coupling agent. Final acidic hydrolysis of the Boc-protecting groups gave the target compounds 3-5 as hydrochloride salts.

As summarized in Table 1, the target compounds 3-5 as well as the reference compounds Vorinostat and Tranylcypromine were profiled for their HDAC1-CoREST3 and LSD1-CoREST3 inhibitory activities in vitro. Unfortunately, compound $\mathbf{5}$ was endowed with poor solubility in the media and, therefore, could not be evaluated. Compounds $\mathbf{3}$ and $\mathbf{4}$ retain the activity of the parent compounds being both active in the nanomolar and in the micromolar range of concentrations against HDAC1 and LSD1, respectively. However, by a closer look to the HDAC1 inhibitory activity, it appears that introduction of a polyamine chain and a second pharmacophore in the para position of the aromatic ring induces a slightly decrease in the activity compared to Vorinostat. Indeed, compounds $\mathbf{3}$ and $\mathbf{4}$ are respectively 9 and 14 - fold less active than Vorinostat $(3: \mathrm{Ki}=$ $26.87 \pm 7.87 \mathrm{nM} ; 4: \mathrm{Ki}=42.52 \pm 8.94 \mathrm{nM}$; Vorinostat: $\mathrm{Ki}=3.04 \pm$ $0.6 \mathrm{nM}$ ). An opposite trend was observed considering the inhibitory activity towards LSD1; in this case, the introduction of the polyamine chain and a second pharmacophore has a positive effect on the inhibitory activity with compounds 3 and $\mathbf{4}$ being respectively 37 and 23 -fold more active than Tranylcypromine (3: $\mathrm{IC}_{50}$ $=2.40 \mu \mathrm{M} ; 4: \mathrm{IC}_{50}=3.85 \mu \mathrm{M}$; Tranylcypromine: $\left.\mathrm{IC}_{50}=89.08 \mu \mathrm{M}\right)$.

Compounds $\mathbf{3}$ and $\mathbf{4}$ were then evaluated in cell-based assay. First, compounds 3 and 4, together with Vorinostat and Tranylcypromine, were evaluated for their cytotoxic activity in MCF7 breast cancer cell line. Vorinostat induced a dose-dependent decrease in cell viability with an $\mathrm{IC}_{50}$ of $38.2 \mu \mathrm{M}$ (data not shown). Higher concentrations of Tranylcypromine were necessary to decrease MCF-7 viability and the concentration required to reach the $\mathrm{IC}_{50}$ value was $2518.9 \mu \mathrm{M}$ (data not shown).

Compounds $\mathbf{3}$ and $\mathbf{4}$ significantly decreased MCF-7 viability starting from the lowest tested concentration and in a dose-dependent manner. The concentration required to reach the $\mathrm{IC}_{50}$ value was $60.2 \mu \mathrm{M}$ for compound 3 and $39.6 \mu \mathrm{M}$ for compound 4 (Fig. 3). The cytotoxic activity of compound $\mathbf{4}$ was similar to that of Vorinostat: at $4.0 \mu \mathrm{M}$, Vorinostat and compound 4 induced $20.8 \%$ and $16.3 \%$ decrease in cell viability, respectively, compared to control cells. Increasing treatment concentrations showed an analogue trend. A $49.3 \%$ and $52.7 \%$ decrease in cell viability was recorded after $24 \mathrm{~h}$ incubation with either Vorinostat or compound 4 at $16.0 \mu \mathrm{M}$ and $35.0 \mu \mathrm{M}$, respectively. At the highest concentration tested $(70.0 \mu \mathrm{M}), 4$ induced a more pronounced cytotoxic 

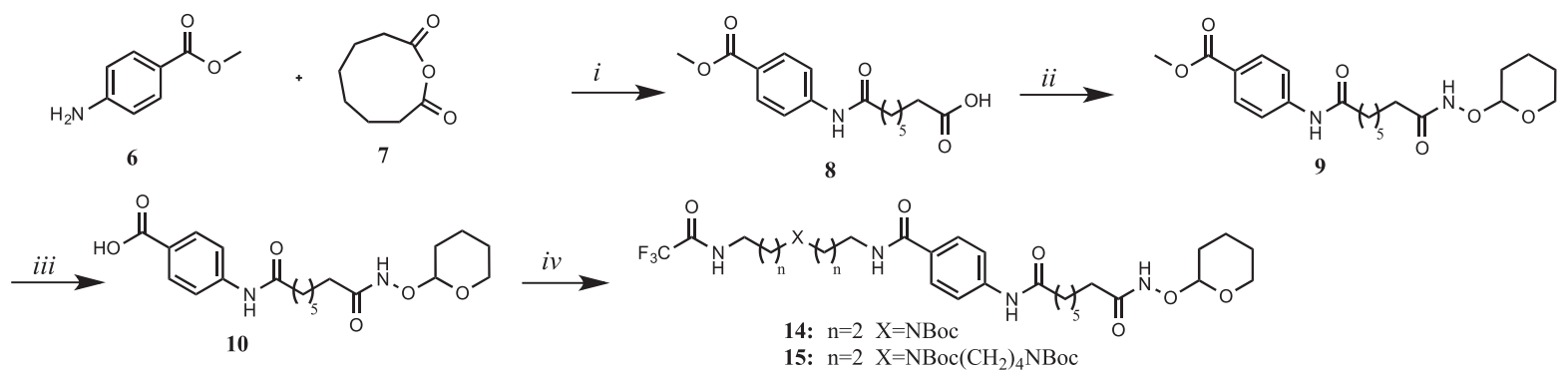

16: $\mathrm{n}=5 \quad \mathrm{X}=\mathrm{NBoc}\left(\mathrm{CH}_{2}\right)_{8} \mathrm{NBoc}$
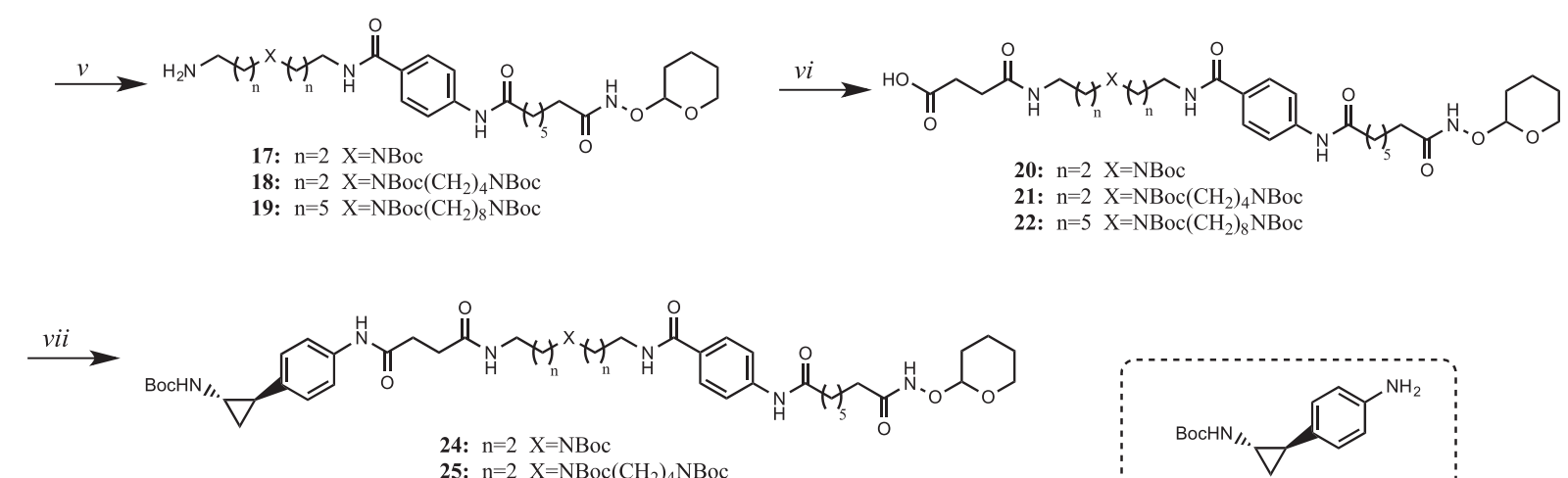

25: $\mathrm{n}=2 \quad \mathrm{X}=\mathrm{NBoc}\left(\mathrm{CH}_{2}\right)_{4} \mathrm{NBoc}$

26: $\mathrm{n}=5 \quad \mathrm{X}=\mathrm{NBoc}\left(\mathrm{CH}_{2}\right)_{8} \mathrm{NBoc}$
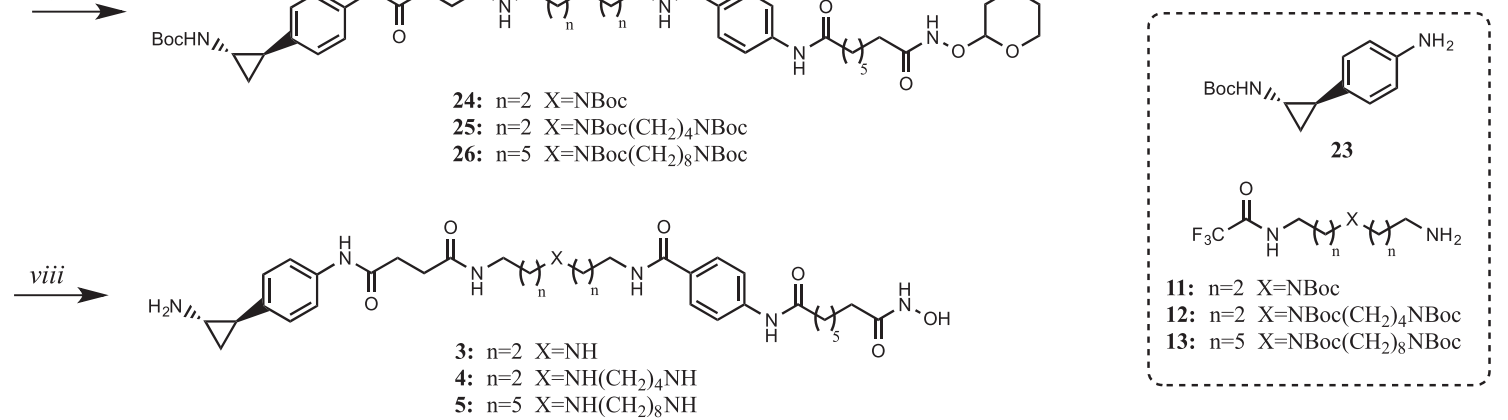

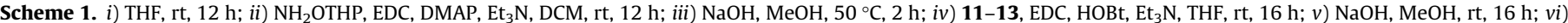
succinic anhydride, $\mathrm{Et}_{3} \mathrm{~N}, \mathrm{DCM}, \mathrm{rt}, 16 \mathrm{~h}$; vii) 23, EDC, DMAP, DCM, rt, 24 h; viii) HCl $4 \mathrm{M} /$ dioxane, DCM rt, 12 h.

Table 1

HDAC1-CoREST3 and LSD1-COREST3 inhibiting activity of the dual binding agents 3-5 in comparison with Vorinostat and Tranylcypromine.

\begin{tabular}{lll}
\hline Compound $^{\mathrm{a}}$ & HDAC1-CoREST3 & $\begin{array}{l}\text { LSD1-CoREST3 } \\
\mathrm{IC}_{50}(\mu \mathrm{M})\end{array}$ \\
\hline $\mathbf{3}$ & Ki $(\mathrm{nM})$ & 2.40 \\
$\mathbf{4}$ & $26.87 \pm 7.87$ & 3.85 \\
$\mathbf{5}$ & $42.52 \pm 8.94$ & n.d. \\
Vorinostat & n.d. & n.d. \\
Tranylcypromine & $3.04 \pm 0.6$ & 89.08 \\
\hline
\end{tabular}

n.d. $=$ not determined .

a Compounds as hydrochloride salts.

effect than Vorinostat (68.6\% vs $56.6 \%$ of dead cells, respectively). Compound 3 exhibited a lower cytotoxic activity with only 52\% decrease in cell viability recorded at the highest concentration tested (Fig. 3). Tranylcypromine showed cytotoxic effects at concentrations much higher than the other compounds (data not shown).

To explore the ability of compounds $\mathbf{3}$ and $\mathbf{4}$ to inhibit HDACs in cell, nuclear extracts of treated MCF-7 cells were compared to Vorinostat plus Tranylcypromine-treated cells. Based on the results of the preliminary evaluation that allowed to define the cytotoxic activity, cells were treated with concentrations similar or lower than the $\mathrm{IC}_{50}$ : $70.0 \mu \mathrm{M}$ for compound $\mathbf{3}, 17.0 \mu \mathrm{M}$ for compound $\mathbf{4}$, a)

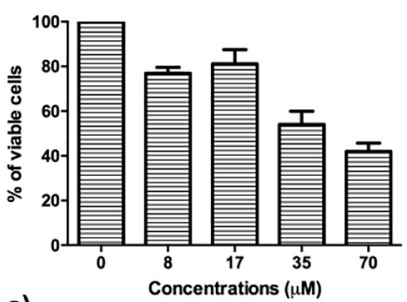

b)

c)
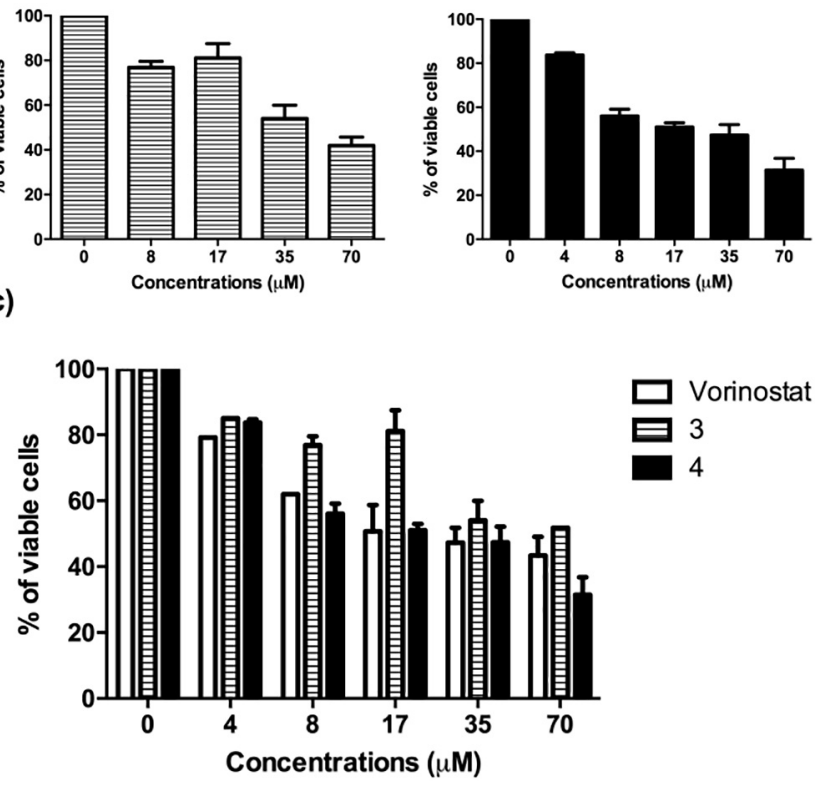

Fig. 3. Cell viability after 24 h treatment of MCF-7 cells with a) compound 3, b) compound $\mathbf{4}$ and c) compounds $\mathbf{3}$ and $\mathbf{4}$ in comparison with Vorinostat. 


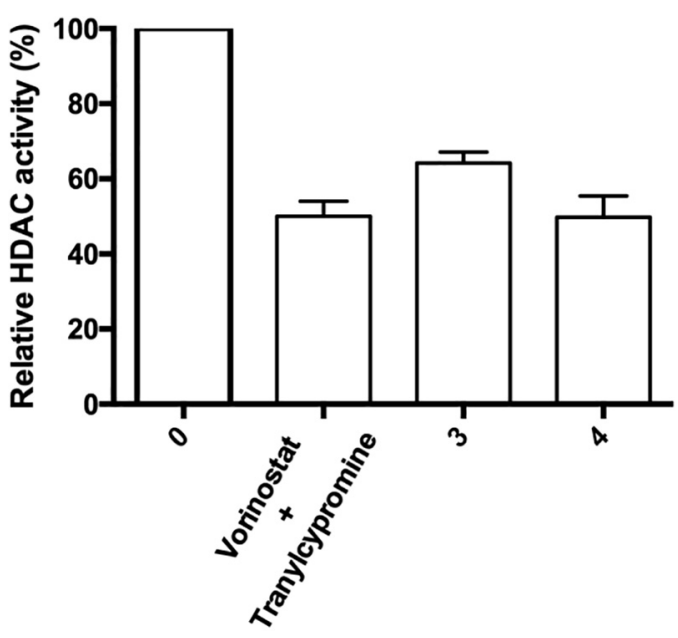

Fig. 4. Relative HDAC activity of Vorinostat $(35 \mu \mathrm{M})$ plus Tranylcypromine (1000 $\mu \mathrm{M})$, compounds $3(70 \mu \mathrm{M})$ and $4(17 \mu \mathrm{M})$ on MCF-7 nuclear extracts.

Vorinostat and Tranylcypromine 35 and $1000.0 \mu \mathrm{M}$, respectively. The HDAC activity levels of all tested compounds were significantly lower than the HDAC activity levels of untreated samples (Fig. 4). A HDAC enzyme inhibition of $50.2 \%$ was recorded after treatment with $17.0 \mu \mathrm{M}$ of compound 4 (Fig. 4). The inhibitory activity of compound $\mathbf{4}$ was similar to that induced by the combination of Vorinostat plus Tranylcypromine (50.0\%). As expected from the cytotoxic results, compound $\mathbf{3}$ had the lowest rate of HDAC inhibition: after $70.0 \mu \mathrm{M}$ treatment, the reduction of enzyme activity was $35.8 \%$ (Fig. 4).

Designing dual binding agents is a well established strategy in medicinal chemistry to provide new drug candidates or biochemical tools. In the present investigation, we explored the structureactivity relationships data available for HDAC and LSD1 inhibitors to design the first class of polyamine-based dual HDAC/LSD1 inhibitors. Indeed, it is well known that HDAC1/2 and LSD1 are associated within the same complex with CoREST. These new dual binding agents were obtained by linking the structure of two well-known HDAC and LSD1 inhibitors, namely Vorinostat and Tranylcypromine. As linker different polyamine chains have been choose. Indeed, polyamines are known to establish additional interaction with negative charged amino acids. The obtained compounds showed in vitro activity towards HDAC1 and LSD1 comparable to that exhibited by the parent compounds Vorinostat and Tranylcypromine as well as in cell HDAC inhibition comparable to that induced by a combination of Vorinostat and Tranylcypromine.

Furthermore, compounds $\mathbf{3}$ and $\mathbf{4}$ possess cytotoxic activity in the micromolar range of concentration in MCF7 breast cancer line. Therefore, this report provides the first polyamine-based compounds that allowed the simultaneous modulation of HDAC and LSD1 with a single molecule. Deeper biological investigations, i.e. antiproliferative effects on other cancer cell lines, analysis of the mechanisms of cell death etc., as well as synthesis of other analogues are currently pursued in our laboratories and will be reported in due course.

\section{Acknowledgments}

This work has been supported by RFO-University of Bologna. AM thanks Mr. Mahmoud Haji for proofreading the manuscript.

\section{A. Supplementary data}

Supplementary data associated with this article can be found, in the online version, at https://doi.org/10.1016/j.bmcl.2018.02.034.

\section{References}

1. You JS, Jones PA. Cancer genetics and epigenetics: two sides of the same coin? Cancer Cell. 2012;22:9-20.

2. Kouzarides T. Chromatin modifications and their function. Cell. 2007; 128:693-705.

3. Yang XJ, Seto E. HATs and HDACs: from structure, function and regulation to novel strategies for therapy and prevention. Oncogene. 2007;26:5310-5318.

4. Weichert W. HDAC expression and clinical prognosis in human malignancies. Cancer Lett. 2009;280:168-176.

5. Bolden JE, Peart MJ, Johnstone RW. Anticancer activities of histone deacetylase inhibitors. Nat Rev Drug Discov. 2006;5:769-784.

6. Benedetti R, Conte M, Altucci L. Targeting histone deacetylases in diseases: where are we? Antioxid Redox Signal. 2015;23:99-126.

7. Mottamal M, Zheng S, Huang TL, Wang G. Histone deacetylase inhibitors in clinical studies as templates for new anticancer agents. Molecules. 2015;20:3898-3941.

8. Shen S, Kozikowski AP. Why hydroxamates may not be the best histone deacetylase inhibitors-what some may have forgotten or would rather forget? ChemMedChem. 2016;11:15-21.

9. Catalano MG, Fortunati N, Pugliese M, et al. Valproic acid, a histone deacetylase inhibitor, enhances sensitivity to doxorubicin in anaplastic thyroid cancer cells. I Endocrinol. 2006;191:465-472.

10. Kim MS, Blake M, Baek JH, Kohlhagen G, Pommier Y, Carrier F. Inhibition of histone deacetylase increases cytotoxicity to anticancer drugs targeting DNA. Cancer Res. 2003;63:7291-7300.

11. Bruzzese F, Leone A, Rocco M, et al. HDAC inhibitor vorinostat enhances the antitumor effect of gefitinib in squamous cell carcinoma of head and neck by modulating ErbB receptor expression and reverting EMT. J Cell Physiol. 2011;226:2378-2390.

12. Nakagawa T, Takeuchi S, Yamada T, et al. EGFR-TKI resistance due to BIM polymorphism can be circumvented in combination with HDAC inhibition. Cancer Res. 2013;73:2428-2434.

13. Fiskus W, Sharma S, Shah B, et al. Highly effective combination of LSD1 (KDM1A) antagonist and pan-histone deacetylase inhibitor against human AML cells. Leukemia. 2014;28:2155-2164.

14. Singh MM, Manton CA, Bhat KP, et al. Inhibition of LSD1 sensitizes glioblastoma cells to histone deacetylase inhibitors. Neuro Oncol. 2011;13:894-903.

15. Hou H, Yu H. Structural insights into histone lysine demethylation. Curr Opin Struct Biol. 2010;20:739-748.

16. Pedersen MT, Helin K. Histone demethylases in development and disease Trends Cell Biol. 2010;20:662-671.

17. You A, Tong JK, Grozinger CM, Schreiber SL. CoREST is an integral component of the CoREST- human histone deacetylase complex. Proc Natl Acad Sci U S A. 2001;98:1454-1458.

18. Palermo G, Minniti E, Greco ML, et al. An optimized polyamine moiety boosts the potency of human type II topoisomerase poisons as quantified by comparative analysis centered on the clinical candidate F14512. Chem Commun (Camb). 2015;51:14310-14313.

19. Minarini A, Milelli A, Tumiatti V, Rosini M, Bolognesi ML, Melchiorre C. Synthetic polyamines: an overview of their multiple biological activities. Amino Acids. 2010;38:383-392.

20. Varghese S, Senanayake T, Murray-Stewart T, et al. Polyaminohydroxamic acids and polyaminobenzamides as isoform selective histone deacetylase inhibitors. $J$ Med Chem. 2008;51:2447-2456.

21. Zhu Q, Huang Y, Marton LJ, Woster PM, Davidson NE, Casero RA. Polyamine analogs modulate gene expression by inhibiting lysine-specific demethylase 1 (LSD1) and altering chromatin structure in human breast cancer cells. Amino Acids. 2012;42:887-898.

22. Pasini A, Marchetti C, Sissi C, Cortesi M, Giordano E, Minarini A, Milelli A. Novel polyamine-naphthalene diimide conjugates targeting histone deacetylases and DNA for cancer phenotype reprogramming. ACS Med Chem Lett. 2017;8 (12):1218-1223.

23. Duan YC, Ma YC, Qin WP, et al. Design and synthesis of tranylcypromine derivatives as novel LSD1/HDACs dual inhibitors for cancer treatment. Eur J Med Chem. 2017;140:392-402.

24. Kalin JH, Wu M, Gomez AV, et al. Targeting the CoREST complex with dual histone deacetylase and demethylase inhibitors. Nat Commun. 2018;9:53.

25. Bonaiuto E, Milelli A, Cozza G, et al. Novel polyamine analogues: from substrates towards potential inhibitors of monoamine oxidases. Eur J Med Chem. 2013;70:88-101.

26. Marchetti C, Minarini A, Tumiatti V, et al. Macrocyclic naphthalene diimides as G-quadruplex binders. Bioorg Med Chem. 2015;23:3819-3830.

27. Binda C, Valente S, Romanenghi M, et al. Biochemical, structural, and biological evaluation of tranylcypromine derivatives as inhibitors of histone demethylases LSD1 and LSD2. J Am Chem Soc. 2010;132:6827-6833. 